\title{
Gradhiva
}

GRADHIV

Revue d'anthropologie et d'histoire des arts

27 | 2018

Sur le vif. Photographie et anthropologie

\section{Anna Lowenhaupt Tsing, Le Champignon de la fin du monde: sur la possibilité de vivre dans les ruines du capitalisme}

Paris, La Découverte/Les Empêcheurs de penser en rond, 2017

Frédéric Keck

\section{CpenEdition}

\section{Journals}

Édition électronique

URL : http://journals.openedition.org/gradhiva/3583

DOI : 10.4000/gradhiva.3583

ISSN : $1760-849 x$

Éditeur

Musée du quai Branly Jacques Chirac

Édition imprimée

Date de publication : 23 mai 2018

Pagination : 258-259

ISBN : 978-2-35744-110-1

ISSN : 0764-8928

\section{Référence électronique}

Frédéric Keck, «Anna Lowenhaupt Tsing, Le Champignon de la fin du monde : sur la possibilité de vivre dans les ruines du capitalisme », Gradhiva [En ligne], 27 | 2018, mis en ligne le 23 mai 2018, consulté le 06 janvier 2021. URL : http://journals.openedition.org/gradhiva/3583 ; DOI : https://doi.org/10.4000/ gradhiva.3583

Ce document a été généré automatiquement le 6 janvier 2021.

(c) musée du quai Branly 


\section{Anna Lowenhaupt Tsing, Le Champignon de la fin du monde: sur la possibilité de vivre dans les ruines du capitalisme}

Paris, La Découverte/Les Empêcheurs de penser en rond, 2017

Frédéric Keck

\section{RÉFÉRENCE}

Anna Lowenhaupt Tsing, Le Champignon de la fin du monde : sur la possibilité de vivre dans les ruines du capitalisme, trad. de l'anglais par Philippe Pignarre, préf. par Isabelle Stengers, Paris, La Découverte/Les Empêcheurs de penser en rond, 2017, 415 p. 


\section{Le champignon de la fin du monde}

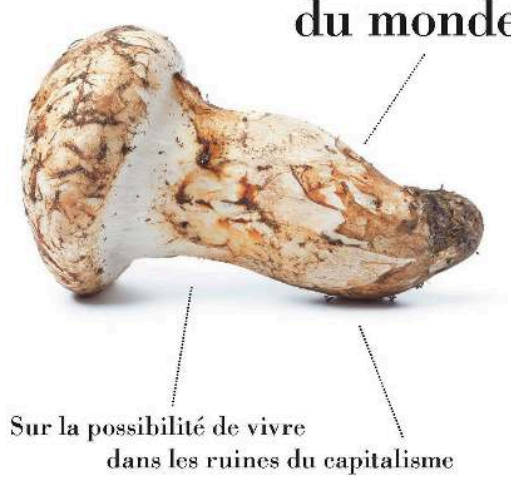

LES EMPECHEURS DE PENSER
EN ROND / LA DECOUVERTE

1 La première phrase de ce très beau livre aurait pu être écrite par Claude Lévi-Strauss : "Que faire quand votre monde commence par s'effondrer? Moi, je pars me promener, et, si j'ai vraiment de la chance, je trouve des champignons.» (p. 31) Lévi-Strauss, luimême grand amateur et collecteur de champignons, s'était intéressé en 1958 à la division du monde entre "mycophiles » (les Slaves et l'Europe méditerranéenne) et «mycophobes» (les Anglo-Saxons), dont il rêvait de faire une clé d'entrée dans la bipolarité du monde sous la guerre froide. Il concluait : « Le parti des hommes pour ou contre les champignons (qui subsistent dans l'économie moderne, comme un des derniers produits sauvages objet de collecte et de ramassage) n'est pour l'humanité qu'une des façons, moins insignifiante qu'il ne semble, de choisir et d'exprimer le type de rapports qu'elle entretient avec la nature, et le monde1.» Soixante ans plus tard, sans le citer, Anna Tsing accomplit ce rêve de Claude Lévi-Strauss. Mais elle opère, ce faisant, une singulière réorientation du monde par rapport à l'Europe, encore privilégiée par Lévi-Strauss. Ce qu'elle interroge, c'est la trajectoire d'un champignon japonais, le matsutake, qui, pour des raisons économiques et écologiques, est aujourd'hui ramassé par des travailleurs laotiens dans le nord-ouest de l'Amérique. C'est ainsi un autre rêve de Lévi-Strauss qu'elle accomplit: celui d'une perspective décentrée sur l'histoire occidentale du capitalisme depuis le détroit de Béring, où humains et non-humains circulent entre l'Asie et l'Amérique.

Le projet d'Anna Tsing est d'interroger «la possibilité de vivre dans les ruines du capitalisme » à partir des relations que les hommes nouent avec cet être apparemment microscopique et insignifiant qu'est le matsutake. Le titre, Le Champignon de la fin du monde, joue sur plusieurs sens. Il désigne d'abord l'effondrement d'un monde social stable, celui du capitalisme industriel, du progrès scientifique et de l'État-providence, au profit de nouveaux rapports économiques caractérisés par la précarité et d'une 
nouvelle situation écologique marquée par la perturbation. "Quand, en 1991, l'Union soviétique s'est effondrée, écrit Anna Tsing, j'ai lu que des milliers de Sibériens, soudain privés des garanties de l'État, s'étaient rués dans les forêts cueillir des champignons. » (p. 32) Mais la fin du monde, c'est aussi la disparition possible de toute vie humaine pensée depuis cet Extrême-Orient qu'a longtemps constitué le Japon pour l'anthropologie européenne. "Le champignon de la fin du monde ", c'est donc à la fois le champignon atomique de Hiroshima - métaphore du désastre à laquelle l'anthropologie ne cesse de revenir depuis 1945 - et les champignons bien réels qui poussent sur les ruines des forêts japonaises. «Quand, en 1945, Hiroshima fut détruite par une bombe atomique, il a été rapporté que la première créature vivante à émerger dans le paysage désolé était un champignon matsutake. » (p. 33)

3 L'anthropologie de la mondialisation proposée par Anna Tsing joue donc sur des effets d'échelle en liant la culture locale des champignons avec des événements globaux comme la fin du capitalisme industriel ou l'apocalypse nucléaire. Mais c'est précisément pour interroger ce qui résiste au changement d'échelle, ce qu'elle appelle «l'inscalabilité ». En ce sens, le champignon matsutake est l'opposé de la canne à sucre, cette plante dont la culture et la consommation standardisées ont favorisé l'essor du capitalisme européen ${ }^{2}$. Le matsutake reste profondément enraciné dans le complexe rhizomatique d'êtres où il émerge, ce qu'Anna Tsing appelle à la suite de Gilles Deleuze " un agencement ", puisqu'il pousse à l'ombre des pins qui profitent eux-mêmes du sol minéral et ensoleillé laissé par la déforestation. Sa transplantation a toujours échoué, ce qui explique qu'il est attaché à un art de cueillir et de cuisiner dont les Japonais restent nostalgiques. Avec l'urbanisation du Japon, cependant, et l'abandon des pratiques paysannes au profit d'une sylviculture industrielle, les matsutake se sont raréfiés, ce qui a obligé les amateurs de ce champignon, dont l'odeur affole ceux qui en sont friands, à se tourner vers des fournisseurs étrangers. Un marché mondial du matsutake s'est alors mis en place, dont Anna Tsing interroge les conditions de production, d'échange et de consommation. Il s'agit de suivre le champignon aux différentes étapes de sa transformation de bien en marchandise, selon les méthodes de l'anthropologie des marchés, en étant attentif pour chaque étape à ce qui résiste à la standardisation : la passion du collecteur, la traduction entre négociateurs, le goût du consommateur ${ }^{3}$.

4 La deuxième partie du livre est consacrée à ce travail de transformation par les marchés, qu'Anna Tsing appelle " accumulation par captation ", au sens où elle permet au capitalisme de produire de la valeur à partir de ce qui émerge en dehors de lui. L'anthropologue décrit les pratiques de cueillette des matsutake par des réfugiés laotiens dans l'État de l'Oregon au nord-ouest des États-Unis. Ces réfugiés avaient combattu l'armée vietnamienne aux côtés des Américains, ce qui les conduit aujourd'hui à revendiquer un mode de vie au nom de "la liberté", même s'ils ne donnent pas à ce terme le même sens que les vétérans blancs de la guerre du Vietnam, avec lesquels il leur arrive de rivaliser dans l'exploration des forêts de pins. « Fiévreuse, la cueillette échappe à la séparation entre les personnes et les choses, si chère à la production industrielle. Les champignons, là, ne se plient pas encore au cortège de marchandises aliénées; ils sont des effets de la liberté des cueilleurs. Et malgré tout cela, cette scène ne peut exister que parce que cette expérience à double visage est poursuivie dans un étrange type de commerce. Les acheteurs, pour convertir les trophées de la liberté en biens commerciaux, orchestrent de manière tout à fait spectaculaire la " concurrence du libre marché " sur des théâtres en plein air. » (p. 132) 
Avec leurs récits de fantômes et leurs pratiques de chasse, les réfugiés laotiens sont plus proches des Amérindiens en cours de disparition que du modèle dominant du consommateur américain, auquel cherchent à s'assimiler au contraire les Japonais arrivés avant la guerre. Anna Tsing, elle-même issue d'une famille de réfugiés chinois, confesse son propre tiraillement entre ces "deux sortes d'Américains asiatiques" (p. 161-162).

5 Les producteurs et les consommateurs de matsutake ont en commun de considérer le champignon comme un don: les premiers parce qu'ils en font un trophée de leur liberté, les seconds parce qu'ils y voient le signe de pratiques aristocratiques passées. Entre ces deux pôles, les champignons deviennent des marchandises par l'effet de l'investissement des Américains et des Japonais dans des efforts de traduction, dont Anna Tsing décrit les aspects à la fois économiques et scientifiques. En une quinzaine de pages éblouissantes (p. 174-188), Anna Tsing retrace les « relations entre capital étatsunien et japonais » pour montrer « qu'elles ont signé la fin des espoirs dans le progrès, compris comme la possibilité d'une amélioration collective » (p.174), puisqu'elles ont mis en place un système concurrentiel de sous-traitance avec des effets ravageurs sur l'environnement social et écologique. La notion de traduction revient à la fin du livre (p. 316) pour désigner cette fois la science des matsutake et la sylviculture, dont elle remarque qu'elle n'a pas pris pour modèle le savoir développé au Japon depuis des siècles, mais s'est constituée en science universelle pour accompagner la circulation des marchandises. Si les études japonaises sur le matsutake étaient considérées par les scientifiques américains comme trop «descriptives ", c'est au contraire ce qui en fait l'intérêt aux yeux de l'anthropologue, qui s'attache à contraster les valeurs opposées du geste de ratisser dans les sylvicultures japonaise et américaine (p. 327-329) ou à suivre les spéculations des généticiens sur les circulations des spores de matsutake entre les deux bords du détroit de Béring (p. 343).

6 Les analyses des traductions entre le Japon et l'Amérique par l'activité capitaliste et la recherche scientifique sont entrecoupées d'un interlude et de quatre chapitres qui promènent le lecteur dans les forêts de l'Oregon, du Yunnan, de Finlande et du Japon, où Anna Tsing retrace les transformations de la forêt pour interroger les possibilités de vie du matsutake. Ces chapitres résultent du projet collaboratif « Matsutake Worlds ", qui a réuni une dizaine de chercheurs depuis 2004, et qui donnera lieu à la publication d'une série de livres dans les années à venir ${ }^{4}$. Il est en effet difficile de mener seul un projet d'anthropologie de la mondialisation par un champignon aussi «insignifiant » que le matsutake. La force du livre d'Anna Tsing qui inaugure cette série est de dessiner un paysage théorique au croisement de l'anthropologie économique de la mondialisation et de l'histoire environnementale des sciences, avec des notions fortes comme « accumulation par captation » ou " communs latents» (p. 369), tout en faisant sentir la valeur d'une ethnographie multi-espèce, attentive à la diversité des êtres qui se déploie sur chaque terrain. À la manière du champignon qu'elle traque, Anna Tsing développe dans ce livre une écriture rhizomatique qui contamine les formes de démonstration de l'anthropologie économique. "Si nous cédons à leur attractivité fongique, les matsutake peuvent nous obliger à faire preuve d'une curiosité qui me semble être la première condition d'une survie collaborative dans les temps précaires. » (p. 32) 


\section{NOTES}

1. Claude Lévi-Strauss, "Dis-moi quels champignons... », La lettre du Collège de France [en ligne], hors-série 2, disponible sur : http://journals.openedition.org/lettre-cdf/222 (consulté le 19 mars 2018). Il s'agit d'une recension du livre de Valentina P. Wasson and Roebert G. Wasson : Sections from Mushrooms, Russia and History, New York, Pantheon Books, 1957, parue dans L'Express le 10 avril 1958.

2. Voir Sidney W. Mintz, Sucre blanc, misère noire : le goût et le pouvoir, trad. de l'anglais par Rula Ghani, Paris, Nathan, 1991.

3. Une telle méthode avait été adoptée en France pour le marché de la truffe par Michèle de La Pradelle dans Les Vendredis de Carpentras : faire son marché en Provence ou ailleurs, Paris, Fayard, 1995 (bien qu'elle ne soit pas citée par Anna Tsing).

4. Voir Timothy K. Choy, Lieba Faier, Michael J. Hathaway, Miyako Inoue, Shiho Satsuka, Anna Tsing, "A new Form of Collaboration in Cultural Anthropology: Matsutake Worlds ", American Ethnologist, 36 (2), 2009 : 380-403.

\section{AUTEURS}

\section{FRÉDÉRIC KECK}

Frederic.KECK[at]quaibranly.fr 